\title{
EDITORIAL
}

\section{PALABRAS DE LA VICEMINISTRA DE SALUD MARTHA LUCIA OSPINA EN LA CEREMONIA INAUGURAL}

Ante todo un cordial saludo de la señora Ministra de Salud, Beatriz Londoño. Estamos muy complacidos con la realización de este 1er. encuentro de semilleros de investigación de las facultades de medicina organizado por la Fundación Universitaria de Ciencias de la Salud.

Es satisfactorio ver como ante la convocatoria respondieron 40 facultades de medicina con 240 proyectos que van desde la investigación básica hasta la clínica y aplicada, sin olvidar la epidemiología y el campo de la salud pública. Promover la investigación, sobre todo aquella aplicada que contribuya a las soluciones que necesita el país, es un objetivo claro del Ministerio de Salud y Protección Social.

A través del Viceministerio de Salud y los Servicios de Salud se está desarrollando un plan de trabajo lleno de grandes retos (independiente de las medidas coyunturales que seguramente han oído). Este plan contiene cinco líneas de acción, las cuales a su vez tienen cada una entre cinco y siete proyectos fundamentales. Las grandes líneas son: 1) salud pública orientada por el estado, 2) atención primaria en salud, 3) fortalecimiento del recurso humano en salud, 4) política antifraude y 5) gestión de riesgo en servicios de salud.

La primera incluye: 1) construcción del plan decenal de salud pública, 2) el fortalecimiento de la promoción de la salud, 3 ) la reconversión de los programas a EGI (estrategias), 4) comunicación en salud-mercadeo social, 5) fortalecimiento del sistema de vigilancia en salud pública y 6) la gestión del conocimiento en salud con el desarrollo de la biblioteca, fortalecimiento del sistema de información y la creación del comité de investigaciones con la coordinación de los estudios y encuestas que realiza o lidera el Ministerio.

En curso hay en la actualidad más de treinta estudios con diferentes grados de avance. Abarcan temas como:
- Nutrición (consumo de sal, encuesta nacional de situación nutricional, consumo de frutas y verduras, etc).

- Salud mental.

- Envejecimiento poblacional.

- Problemas de salud (parasitismo intestinal, mortalidad infantil, TBC).

- Salud sexual y reproductiva.

- Salud oral.

- Factores de riesgo (encuesta mundial de tabaquismo, sustancias psioactivas, etc).

- Calidad y oferta de los servicios de salud.

- Medio ambiente (mercurio, calidad de aire).

- Calidad de vida.

- Violencia.

- Estos estudios se realizan en alianza con importantes instituciones como: DNP, DANE, Profamilia, ICBF y Universidades (Nacional, Antioquia, Valle, Andes, Externado). Se efectúan en cooperación con organizaciones internacionales reconocidas como OMS, CDC, OPS y otras. Además hacemos parte del panel de evaluación de Colciencias y a través de ellas, sacamos convocatorias para las GAI e hicimos con ellos la agenda de investigación (diagnóstico por territorios sobre los intereses o temas de probable investigación).

Con este contexto queda claro que estamos muy interesados en conocer las propuestas de investigación que hoy nos presentarán las facultades de medicina y queremos ofrecerles la publicación de las iniciativas en la biblioteca virtual de la página del Ministerio.

Muchas gracias. 